International Electronic Journal of Algebra VOLUME 25 (2019) 171-185

DOI: $10.24330 /$ ieja.504142

\title{
STAR OPERATIONS ON KUNZ DOMAINS
}

\author{
Dario Spirito \\ Received: 25 May 2018; Revised: 10 September 2018; Accepted: 19 September 2018
}

Communicated by Abdullah Harmancı

\begin{abstract}
We study star operations on Kunz domains, a class of analytically irreducible, residually rational domains associated to pseudo-symmetric numerical semigroups, and we use them to refute a conjecture of Houston, Mimouni and Park. We also find an estimate for the number of star operations in a particular case, and a precise counting in a sub-case.
\end{abstract}

Mathematics Subject Classification (2010): 13A15, 13E05, 13G05

Keywords: Star operation, pseudo-symmetric semigroup, Kunz domain, star regular domain

\section{Introduction}

Let $D$ be an integral domain with quotient field $K$, and let $\mathcal{F}(D)$ be the set of fractional ideals of $D$, i.e., the set of $D$-submodules $I$ of $K$ such that $x I \subseteq D$ for some $x \in K \backslash\{0\}$.

A star operation on $D$ is a map $\star: \mathcal{F}(D) \longrightarrow \mathcal{F}(D), I \mapsto I^{\star}$, such that, for every $I, J \in \mathcal{F}(D)$ and every $x \in K$ :

- $I \subseteq I^{\star}$

- if $I \subseteq J$, then $I^{\star} \subseteq J^{\star}$;

- $\left(I^{\star}\right)^{\star}=I^{\star}$

- $x \cdot I^{\star}=(x I)^{\star}$;

- $D=D^{\star}$.

A fractional ideal $I$ is $\star$-closed if $I=I^{\star}$.

The easiest example of a non-trivial star operation is the v-operation $v: I \mapsto$ $(D:(D: I))$, where if $I, J \in \mathcal{F}(D)$ we define $(I: J):=\{x \in K \mid x J \subseteq I\}$. An ideal that is $v$-closed is said to be divisorial; if $I$ is divisorial and $\star$ is any other star operation then $I=I^{\star}$. We denote by $d$ the identity, which is obviously a star operation.

Recently, the cardinality of the set $\operatorname{Star}(D)$ of the star operations on $D$ has been studied, especially in the case of Noetherian [4,8] and Prüfer domains [3,5]. In particular, Houston, Mimouni and Park started studying the relationship between 
the cardinality of $\operatorname{Star}(D)$ and the cardinality of $\operatorname{Star}(T)$, where $T$ is an overring of $D$ (an overring of $D$ is a ring comprised between $D$ and $K$ ) [6,7]: they called a domain star regular if $|\operatorname{Star}(D)| \geq|\operatorname{Star}(T)|$ for every overring of $T$. While even simple domains may fail to be star regular (for example, there are domains with just one star operation having an overring with infinitely many star operations [6, Example 1.3]), they conjectured that every one-dimensional local Noetherian domain $D$ such that $1<|\operatorname{Star}(D)|<\infty$ is star regular, and proved it when the residue field of $D$ is infinite [6, Corollary 1.18].

In this context, a rich source of examples are semigroup rings, that is, subrings of the power series ring $K[[X]]$ (where $K$ is a field, usually finite) of the form $K[[S]]:=$ $K\left[\left[X^{S}\right]\right]:=\left\{\sum_{i} a_{i} X^{i} \mid a_{i}=0\right.$ for all $\left.i \notin S\right\}$, where $S$ is a numerical semigroup (i.e., a submonoid $S \subseteq \mathbb{N}$ such that $\mathbb{N} \backslash S$ is finite). Star operations can also be defined on numerical semigroups [14], and there is a link between star operations on $S$ and star operations on $K[[S]$ ]: for example, every star operation on $S$ induces a star operation on $K[[S]]$, and $|\operatorname{Star}(S)|=1$ if and only if $|\operatorname{Star}(K[[S]])|=1$ [14, Theorem 5.3], with the latter result corresponding to the equivalence between $S$ being symmetric and $K[[S]]$ being Gorenstein $[2,10]$. A detailed study of star operations on some numerical semigroup rings was carried out in [15].

In this paper, we study star operations on Kunz domains, which are, roughly speaking, a generalization of rings in the form $K[[S]]$ where $S$ is a pseudo-symmetric semigroup (see the beginning of the next section for the definitions). We show that, if $R$ is a Kunz domain whose residue field is finite and the length of $\bar{R} / R$ is at least 4 (where $\bar{R}$ is the integral closure of $R$ ) then $R$ is a counterexample to HoustonMimouni-Park's conjecture; that is, $R$ satisfies $1<|\operatorname{Star}(R)|<\infty$ but there is an overring $T$ of $R$ with more star operations than $R$. In Section 3, we also study more deeply one specific class of domains, linking the cardinality of $\operatorname{Star}(R)$ with the set of vector subspaces of a vector space over the residue field of $R$, and calculate the cardinality of $\operatorname{Star}(R)$ when the value semigroup of $R$ is $\langle 4,5,7\rangle$.

We refer to [13] for information about numerical semigroups, and to [1] for the passage from numerical semigroups to one-dimensional local domains.

\section{Kunz domains}

A numerical semigroup is a subset $S \subseteq \mathbb{N}$ such that $0 \in S$, that is closed by addition and such that $\mathbb{N} \backslash S$ is finite. If $S$ is a numerical semigroup, we let $g:=g(S):=\sup (\mathbb{Z} \backslash S)$ be the genus of $S$ and $\mu:=\mu(S):=\min (S \backslash\{0\})$ be the multiplicity of $S$. If $a_{1}, \ldots, a_{n}$ are coprime integers, we denote by $\left\langle a_{1}, \ldots, a_{n}\right\rangle$ the 
numerical semigroup generated by $a_{1}, \ldots, a_{n}$, i.e., $\left\langle a_{1}, \ldots, a_{n}\right\rangle=\left\{\lambda_{1} a_{1}+\cdots+\lambda_{n} a_{n} \mid\right.$ $\left.\lambda_{1}, \ldots, \lambda_{n} \in \mathbb{N}\right\}$.

Let $\left(V, M_{V}\right)$ be a discrete valuation ring with associated valuation $\mathbf{v}$. We shall consider local subrings $\left(R, M_{R}\right)$ of $V$ with the following properties:

- $R$ and $V$ have the same quotient field;

- the integral closure of $R$ is $V$;

- $R$ is Noetherian;

- the conductor ideal $(R: V)$ is nonzero;

- the inclusion $R \hookrightarrow V$ induces an isomorphism of residue fields $R / M_{R} \longrightarrow$ $V / M_{V}$.

Equivalently, $R$ is an analytically irreducible, residually rational one-dimensional Noetherian local domain having integral closure $V$. For every such $R$, the set $\mathbf{v}(R):=\{\mathbf{v}(r) \mid r \in R\}$ is a numerical semigroup. We state explicitly a property which we will be using many times.

Proposition 2.1 ([12, Corollary to Proposition 1]). Let $R$ be as above, and let $I \subseteq J$ be R-submodules of the quotient field of $R$. Then,

$$
\ell_{R}(J / I)=|\mathbf{v}(J) \backslash \mathbf{v}(I)|,
$$

where $\ell_{R}$ is the length of an $R$-module.

We say that a numerical semigroup $S$ is pseudo-symmetric if $g=g(S)$ is even and, for every $a \in \mathbb{N}, a \neq g / 2$, either $a \in S$ or $g-a \in S$. Following [1] (and using the characterization in [1, Proposition II.1.12]), we give the following definition.

Definition 2.2. A ring $R$ satisfying the previous conditions is a Kunz domain if $\mathbf{v}(R)$ is a pseudo-symmetric semigroup.

From now on, we suppose that $R$ is a Kunz domain, and we set $g:=g(\mathbf{v}(R))$ and $\tau:=g / 2$. The hypotheses on $R$ guarantee that, if $x \in V$ is such that $\mathbf{v}(x)>g$, then $x \in R$ [10, Theorem, p.749].

Our first stage is constructing an overring $T$ of $R$ which we will use in the counterexample.

Lemma 2.3. Let $y \in V$ be an element of valuation $g$, and let $T:=R[y]$. Then:

(a) $T$ contains all elements of valuation $g$;

(b) $\mathbf{v}(T)=\mathbf{v}(R) \cup\{g\}$;

(c) $\ell_{R}(T / R)=1$;

(d) $T=R+y R$. 
Proof. Let $y^{\prime} \in V$ be another element of valuation $g$. Then, $\mathbf{v}\left(y / y^{\prime}\right)=0$, and thus $c:=y / y^{\prime}$ is a unit of $V$. Hence, there is a $c^{\prime} \in R$ such that the images of $c$ and $c^{\prime}$ in the residue field of $V$ coincide; in particular, $c=c^{\prime}+m$ for some $m \in M_{V}$. Hence,

$$
y^{\prime}=c y=\left(c^{\prime}+m\right) y=c^{\prime} y+m y .
$$

Since $c^{\prime} \in R$, we have $c^{\prime} y \in R[y]$; furthermore, $\mathbf{v}(m y)=\mathbf{v}(m)+\mathbf{v}(y)>\mathbf{v}(y)=g$, and thus $m y \in R$. Hence, $y^{\prime} \in R[y]$, and thus $R[y]$ contains all elements of valuation $g$.

The fact that $\mathbf{v}(T)=\mathbf{v}(R) \cup\{g\}$ is trivial; hence, $\ell_{R}(T / R)=|\mathbf{v}(T) \backslash \mathbf{v}(R)|=1$. The last point follows from the fact that $R+y R$ is an $R$-module, from $R \subsetneq R+y R \subseteq$ $T$ and from $\ell_{R}(T / R)=1$.

In particular, the previous proposition shows that $T$ is independent from the element $y$ chosen. From now on, $T$ will always denote this ring.

We denote by $\mathcal{F}_{0}(R)$ the set of $R$-fractional ideals $I$ such that $R \subseteq I \subseteq V$. If $I$ is any fractional ideal over $R$, and $\alpha \in I$ is an element of minimal valuation, then $\alpha^{-1} I \in \mathcal{F}_{0}(R)$; hence, the action of any star operation is uniquely determined by its action on $\mathcal{F}_{0}(R)$. Furthermore, $V^{\star}=V$ for all $\star \in \operatorname{Star}(R)$ (since $(R:(R$ : $V))=V$ ) and thus $I^{\star} \in \mathcal{F}_{0}(R)$ for all $I \in \mathcal{F}_{0}(R)$, i.e., $\star$ restricts to a map from $\mathcal{F}_{0}(R)$ to itself.

To analyze star operations, we want to subdivide them according to whether they close $T$ or not. One case is very simple.

Proposition 2.4. If $\star \in \operatorname{Star}(R)$ is such that $T \neq T^{\star}$, then $\star=v$.

Proof. Suppose $\star \neq v$ : then, there is a fractional ideal $I \in \mathcal{F}_{0}(R)$ that is $\star$-closed but not divisorial. By [1, Lemma II.1.22], $\mathbf{v}(I)$ is not divisorial (in $\mathbf{v}(R)$ ) and thus by [1, Proposition I.1.16] there is a positive integer $n \in \mathbf{v}(I)$ such that $n+\tau \notin \mathbf{v}(I)$.

Let $x \in I$ be an element of valuation $n$, and consider the ideal $J:=x^{-1} I \cap V$ : being the intersection of two $\star$-closed ideals, it is itself $\star$-closed. Since $\mathbf{v}(x)>0$, every element of valuation $g$ belongs to $J$; on the other hand, by the choice of $n$, no element of valuation $\tau$ can belong to $J$.

Consider now the ideal $L:=\left(R: M_{R}\right)$ : then, $L$ is divisorial (since $M_{R}$ is divisorial) and, using [1, Proposition II.1.16(1)],

$$
\mathbf{v}(L)=\left(\mathbf{v}(R)-\mathbf{v}\left(M_{R}\right)\right)=\mathbf{v}(R) \cup\{\tau, g\} .
$$

We claim that $T=J \cap L$ : indeed, clearly $J \cap L$ contains $R$, and if $y$ has valuation $g$ then $y \in J \cap L$ by construction; thus $T=R+y R \subseteq J \cap L$. On the other hand, $\mathbf{v}(J \cap L) \subseteq \mathbf{v}(J) \cap \mathbf{v}(L)=\mathbf{v}(R) \cup\{g\}$, and thus $J \cap L \subseteq T$. 
Hence, $T=J \cap L$; since $J$ and $L$ are both $\star$-closed, so is $T$. Therefore, if $T \neq T^{\star}$ then $\star$ must be the divisorial closure, as claimed.

Suppose now that $T=T^{\star}$. Then, $\star$ restricts to a star operation $\star_{1}:=\left.\star\right|_{\mathcal{F}(T)}$, and the amount of information we lose in the passage from $\star$ to $\star_{1}$ depends on the $R$-fractional ideals that are not ideals over $T$. We can determine them explicitly.

Recall that the canonical ideal of a ring $R$ is a (fractional) ideal $\omega$ such that $(\omega:(\omega: I))=I$ for every fractional ideal $I$. Not every integral domain has a canonical ideal; however, Kunz domains (or, more generally, Noetherian onedimensional local domains whose completion is reduced [11, Korollar 2.12], so in particular domains satisfying the five properties at the beginning of this section) have a canonical ideal; furthermore, if $S$ is a Kunz domain, then an ideal $\omega \in \mathcal{F}_{0}(R)$ is canonical if and only if $\mathbf{v}(\omega)=S \cup\{x \in \mathbb{N} \mid g(S)-x \notin S\}=S \cup\{\tau\}$ [9, Satz 5].

Lemma 2.5. Let $I \in \mathcal{F}_{0}(R), I \neq R$. Then, the following are equivalent.

(i) $\mathbf{v}(I)=\mathbf{v}(R) \cup\{\tau\}$;

(ii) $I$ does not contain any element of valuation $g$;

(iii) $I T \neq I$;

(iv) $I$ is a canonical ideal of $R$.

Furthermore, in this case, there is a unit $u$ of $R$ such that $R \subseteq u I \subseteq\left(R: M_{R}\right)$ and $(u I)^{v}=\left(R: M_{R}\right)$.

Proof. (i) $\Rightarrow$ (ii) is obvious.

(ii) $\Rightarrow$ (iii) Since $R \subseteq I$, there is an element $x$ of $I$ of valuation 0 ; hence, $I T$ contains an element of valuation $g$, and thus $I T \neq I$.

(iii) $\Rightarrow$ (i) Suppose there is an $x \in I$ such that $\mathbf{v}(x) \notin \mathbf{v}(R) \cup\{\tau\}$. Since $\mathbf{v}(R)$ is pseudo-symmetric, there is an $y \in R$ such that $\mathbf{v}(y)=g-\mathbf{v}(x)$; hence, $I$ contains an element (explicitly, $x y$ ) of valuation $g$ and, by the proof of Lemma 2.3, it follows that it contains every element of valuation $g$.

Fix now an element $y \in V$ of valuation $g$. Since $I T \neq I$, there are $i \in I, t \in T$ such that it $\notin I$. By Lemma 2.3(d), there are $r, r^{\prime} \in R$ such that $t=r+y r^{\prime}$; hence, $i t=i\left(r+y r^{\prime}\right)=i r+i y r^{\prime}$. Both $i r$ and $i y r^{\prime}$ are in $I$, the former since it belongs to $I R=I$ and the latter because its valuation is at least $g$. However, this contradicts it $\notin I$; therefore, $\mathbf{v}(I) \subseteq \mathbf{v}(R) \cup\{\tau\}$.

If $\mathbf{v}(I)=\mathbf{v}(R)$, then we must have $I=R$, against our hypothesis; therefore, $\mathbf{v}(I)=\mathbf{v}(R) \cup\{\tau\}$.

(i) $\Leftrightarrow$ (iv) follows from $[9$, Satz 5] and the fact that $R$ is a Kunz domain. 
For the last claim, we first note that $\left(R: M_{R}\right)$ is divisorial (since $M_{R}$ is divisorial). By [1, Proposition II.1.16], $\mathbf{v}\left(\left(R: M_{R}\right)\right)=S \cup\{\tau, g\}$; if $x \in\left(R: M_{R}\right)$ has valuation $\tau$, then $I^{\prime}:=R+x R$ is a canonical ideal (since $\mathbf{v}\left(I^{\prime}\right)=S \cup\{\tau\}$ ) and is contained between $R$ and $\left(R: M_{R}\right)$. Since $R$ is local, and $I$ is a canonical ideal too, there is a unit $u$ of $R$ such that $I^{\prime}=u I$ [11, Satz 2.8(b)]; the claim follows.

Proposition 2.6. The map

$$
\begin{aligned}
\Psi: \operatorname{Star}(R) \backslash\{d, v\} & \longrightarrow \operatorname{Star}(T) \\
\star & \longmapsto \star \mid \mathcal{F}(T)
\end{aligned}
$$

is well-defined and injective.

Proof. By Proposition 2.4, if $\star \neq v$ then $T=T^{\star}$, and thus $\left.\star\right|_{\mathcal{F}(T)}$ is a star operation on $T$; hence, $\Psi$ is well-defined. We claim that it is injective: suppose $\star_{1} \neq \star_{2}$. Then, there is an $I \in \mathcal{F}_{0}(R)$ such that $I^{\star_{1}} \neq I^{\star_{2}}$. If $I$ is a $T$-module then $\Psi\left(\star_{1}\right) \neq \Psi\left(\star_{2}\right)$; suppose $I$ is not a $T$-module.

By Lemma 2.5, $I$ can only be $R$ or a canonical ideal of $R$. In the former case, since $\star_{1}$ and $\star_{2}$ are star operations, $R^{\star_{1}}=R=R^{\star_{2}}$, a contradiction. In the latter case, by multiplying by a unit we can suppose that $I \subseteq\left(R: M_{R}\right)$. Then, $\ell\left(\left(R: M_{R}\right) / I\right)=1$, and thus $I^{\star_{i}}$ can only be $I$ or $\left(R: M_{R}\right)$; suppose now that $I^{\star}=I$ for some $\star \in \operatorname{Star}(R)$. By definition of the canonical ideal, $J=(I:(I: J))$ for every ideal $J$; since $(I: L)$ is always $\star$-closed if $I$ is $\star$-closed, it follows that $\star$ must be the identity. Since $\star_{1}, \star_{2} \neq d$, we must have $I^{\star_{1}}=\left(R: M_{R}\right)=I^{\star_{2}}$, against the assumptions. Thus, $\Psi$ is injective.

An immediate corollary of the previous proposition is that $|\operatorname{Star}(R)| \leq|\operatorname{Star}(T)|+$ 2. Our counterexample thus involves finding star operations of $T$ that do not belong to the image of $\Psi$; to do so, we restrict to the case $\ell_{R}(V / R) \geq 4$ or, equivalently, $|\mathbb{N} \backslash \mathbf{v}(R)| \geq 4$. This excludes exactly two pseudo-symmetric numerical semigroups, namely $\langle 3,4,5\rangle$ and $\langle 3,5,7\rangle$.

Lemma 2.7. Let $S$ be a pseudo-symmetric numerical semigroup, let $g:=\max (\mathbb{N} \backslash$ $S)$ and let $S^{\prime}:=S \cup\{g\}$. If $|\mathbb{N} \backslash S| \geq 4$, then there are $a, b \in\left(S^{\prime}-M_{S^{\prime}}\right) \backslash S^{\prime}, a \neq b$, such that $2 a, 2 b, a+b \in S^{\prime}$.

Proof. Let $\mu$ be the multiplicity of $S$. We claim that $a:=\tau$ and $b:=g-\mu$ are the two elements we are looking for.

Since $a+M_{S} \subseteq S$ and $a+g>g$ (and so $a+g \in M_{S}$ ) we have $a \in\left(S^{\prime}-M_{S^{\prime}}\right)$. Furthermore, since $|\mathbb{N} \backslash S| \geq 4$, we have $g>\mu$, and thus $b+m \geq g$ for all $m \in M_{S^{\prime}}$. 
By the previous point, $a+m, b+m \in S^{\prime} \cup\{a, b\}$ for every $m \in M_{S^{\prime}}$. Since $a=\tau$, we have $2 a=g \in S^{\prime}$.

If $g>2 \mu$, then $a>\mu$, and so $a+b \geq g$, which implies $a+b \in S^{\prime}$; moreover, also $b>\mu$, and thus $2 b=b+b \geq g-\mu+\mu=g$, so that $2 b \in S^{\prime}$.

If $g<2 \mu$, then $g$ must be equal to $2 \mu-2$ or to $\mu-1$; the latter case is impossible since $|\mathbb{N} \backslash S| \geq 4$. Hence, $b=\mu-2$ and $a=\mu-1$. Then, $2 b=2 \mu-4$ and $a+b=2 \mu-3$; again since $|\mathbb{N} \backslash S| \geq 4$, we must have $\mu>3$, and thus $2 b>a+b \geq \mu$. Furthermore, in this case $S^{\prime}=\{0, \mu, \ldots\}$, and so $a+b, 2 b \in S^{\prime}$, as claimed.

Proposition 2.8. Let $K$ be the residue field of $R$, and suppose that $\ell_{R}(V / R) \geq 4$. There are at least $|K|+1$ star operations on $T$ that do not close $\left(R: M_{R}\right)$.

Proof. We first note that $\left(R: M_{R}\right)$ is a $T$-module. Indeed, let $x \in\left(R: M_{R}\right)$ and $t \in T$ : then, $t=r+a y$, with $r, a \in R$ and $\mathbf{v}(y)=g$, and so $x t=x r+a x y$. Both $x r$ and axy belong to $\left(R: M_{R}\right)$, the former because $\left(R: M_{R}\right)$ is an $R$-module and the latter since its valuation is at least $g$ : hence, $x t \in\left(R: M_{R}\right)$. Thus, it makes sense to ask if a star operation on $T$ closes $\left(R: M_{R}\right)$.

Furthermore, $T \subsetneq\left(R: M_{R}\right) \subseteq\left(T: M_{T}\right)$ : the first containment follows from the previous reasoning (and the fact that $\left(R: M_{R}\right)$ contains an element of valuation $\tau$ while $T$ does not). To see the second containment, we note that $M_{T}=M_{R}+\{x \in$ $V \mid \mathbf{v}(x)=g\}$; thus, if $x \in\left(R: M_{R}\right)$ and $y \in M_{T}$, we can write $y=y_{1}+y_{2}$ (with $y_{1} \in M_{R}$ and $\mathbf{v}\left(y_{2}\right)=g$ ) and so $x y=x\left(y_{1}+y_{2}\right)=x y_{1}+x y_{2}$. Now $x y_{1} \in R \subseteq T$, while $\mathbf{v}\left(x y_{2}\right) \geq g$ since $\mathbf{v}(x) \geq 0$, and thus both $x y_{1}$ and $x y_{2}$ belong to $T$. It follows that $x \in\left(T: M_{T}\right)$, i.e., $\left(R: M_{R}\right) \subseteq\left(T: M_{T}\right)$. Therefore, $\left(R: M_{R}\right)^{v_{T}}=\left(T: M_{T}\right)$, where $v_{T}$ is the $v$-operation on $T$.

Let $S^{\prime}:=\mathbf{v}(T)$ : by Lemma 2.7 , we can find $a, b \in\left(S^{\prime}-M_{S^{\prime}}\right) \backslash S^{\prime}$ such that $2 a, 2 b, a+b \in S^{\prime}$. Choose $x, y \in\left(T: M_{T}\right)$ such that $\mathbf{v}(x)=a$ and $\mathbf{v}(y)=b$ (and, without loss of generality, suppose $\left.y \notin\left(R: M_{R}\right)\right)$ : they exist since $\mathbf{v}\left(\left(T: M_{T}\right)\right)=$ $\left(S^{\prime}-M_{S^{\prime}}\right)[1$, Proposition II.1.16].

Let $\left\{\alpha_{1}, \ldots, \alpha_{q}\right\}$ be a complete set of representatives of $R / M_{R}$ (or, equivalently, of $\left.T / M_{T}\right)$. Then, $x+\alpha_{i} y \in\left(T: M_{T}\right)$ for each $i$, and by the choice of $\mathbf{v}(x)$ and $\mathbf{v}(y)$ the module $T_{i}=T+\left(x+\alpha_{i} y\right) T$ is a ring, equal to $T\left[x+\alpha_{i} y\right]$. Define $\star_{i}$ as the star operation

$$
I \mapsto I^{v_{T}} \cap I T_{i} .
$$

We claim that $\star_{i}$ closes $T_{i}$ but not $T_{j}$ for $j \neq i$.

Indeed, clearly $T_{i}^{\star_{i}}=T_{i}$. If $j \neq i$, then $T_{i} T_{j}$ contains both $x+\alpha_{i} y$ and $x+$ $\alpha_{j} y$, and thus it contains their difference $\left(\alpha_{i}-\alpha_{j}\right) y$. Since $\alpha_{i}$ and $\alpha_{j}$ are units corresponding to different residues, it follows that $\alpha_{i}-\alpha_{j}$ is a unit of $R$, and thus 
of $T$; hence, $y \in T_{i} T_{j}$. By construction, $y \in\left(T: M_{T}\right)$ : thus, $y \in T_{i}^{\star_{j}}$. On the other hand, $y \notin T_{i}$, and thus $T_{i}^{\star j} \neq T_{i}$.

Thus, $\left\{\star_{1}, \ldots, \star_{q}\right\}$ are $q=|K|$ different star operations. Furthermore, none of them closes $\left(R: M_{R}\right)$, since

$$
\left(R: M_{R}\right)^{\star_{i}}=\left(T: M_{T}\right) \cap\left(R: M_{R}\right) T\left[x+a_{i} y\right]
$$

contains $y$, while $y \notin\left(R: M_{R}\right)$.

To conclude the proof, it is enough to note that none of the $\star_{i}$ are the divisorial closure (since they close one of the $T_{i}$, none of which is divisorial); thus, adding $v_{T}$ to the $\star_{i}$, we have $q+1$ star operations that do not close $\left(R: M_{R}\right)$.

We are now ready to show that $R$ is the desired counterexample.

Theorem 2.9. Let $R$ be a Kunz domain with finite residue field, and suppose that $\ell_{R}(V / R) \geq 4$. Then, $1<|\operatorname{Star}(R)|<\infty$, but $R$ is not star regular.

Proof. Since $K$ is a finite field and $R$ is not Gorenstein, by [4, Theorem 2.5] $1<|\operatorname{Star}(R)|<\infty$, and the same for $T$.

By Proposition 2.6, we have $|\operatorname{Star}(R)| \leq 2+|\Psi(\operatorname{Star}(R))|$; by Proposition 2.8, we have $|\Psi(\operatorname{Star}(R))| \leq|\operatorname{Star}(T)|-|K|-1$. Hence,

$$
\begin{aligned}
|\operatorname{Star}(R)| & \leq 2+|\operatorname{Star}(T)|-|K|-1= \\
& =|\operatorname{Star}(T)|-|K|+1<|\operatorname{Star}(T)|
\end{aligned}
$$

since $|K| \geq 2$. The claim is proved.

\section{The case $\mathbf{v}(R)=\langle n, n+1, \ldots, 2 n-3,2 n-1\rangle$}

In this section, we specialize to the case of Kunz domains $R$ such that $\mathbf{v}(R)=$ $\langle n, n+1, \ldots, 2 n-1,2 n-3\rangle=\{0, n, n+1, \ldots, 2 n-1,2 n-3, \ldots\}$, where $n \geq 4$ is an integer. It is not hard to see that this semigroup is pseudo-symmetric, with $g=2 n-2$ and $\tau=n-1$.

We note that this semigroup is pseudo-symmetric also if $n=3$, for which the number of star operations has been calculated in [8, Proposition 2.10]: in this case, we have $|\operatorname{Star}(R)|=4$.

By Lemma 2.5, the only $I \in \mathcal{F}_{0}(R)$ such that $I T \neq I$ are $R$ and the canonical ideals. From now on, we denote by $\mathcal{G}$ the set $\left\{I \in \mathcal{F}_{0}(R) \mid I T=I\right\}$; we want to parametrize $\mathcal{G}$ by subspaces of a vector space.

Lemma 3.1. Let $K$ be the residue field of $R$. Then, there is an order-preserving bijection between $\mathcal{G}$ and the set of vector subspaces of $K^{n-1}$. 
Proof. Every $I \in \mathcal{G}$ contains $T$. The quotient of $R$-modules $\pi: V \mapsto V / T$ induces a map

$$
\begin{aligned}
& \tilde{\pi}: \mathcal{G} \longrightarrow \mathcal{P}(V / T) \\
& I \longmapsto \pi(I),
\end{aligned}
$$

where $\mathcal{P}(V / T)$ denotes the power set of $V / T$. It is obvious that $\widetilde{\pi}$ is injective.

The map $\pi$ induces on $V / T$ a structure of $K$-vector space of dimension $n-1$. If $I \in \mathcal{G}$, then its image along $\widetilde{\pi}$ will be a vector subspace; conversely, if $W$ is a vector subspace of $V / T$ then $\pi^{-1}(W)$ will be an ideal in $\mathcal{G}$. The claim is proved.

For an arbitrary domain $D$ and a fractional ideal $I$ of $D$, the star operation generated by $I$ is the map [14, Section 5]

$$
\star_{I}: J \mapsto(I:(I: J)) \cap J^{v}=J^{v} \cap \bigcap_{\gamma \in(I: J) \backslash\{0\}} \gamma^{-1} I ;
$$

this star operation has the property that, if $I$ is $\star$-closed for some $\star \in \operatorname{Star}(D)$ and $J$ is $\star_{I}$-closed, then $J$ is also $\star$-closed. If $\Delta \subseteq \mathcal{F}(D)$, we define $\star_{\Delta}$ as the map

$$
\star_{\Delta}: J \mapsto \bigcap_{I \in \Delta} J^{\star_{I}} .
$$

In the present case, we can characterize when an ideal is $\star_{\Delta}$-closed.

Proposition 3.2. Let $I, J \in \mathcal{G}$ and let $\Delta \subseteq \mathcal{G}$ be a set of nondivisorial ideals.

(a) $I$ is divisorial if and only if $n-1 \in \mathbf{v}(I)$;

(b) $I^{v}=I \cup\{x \mid \mathbf{v}(x) \geq n-1\}$;

(c) if $I, J$ are nondivisorial, then $I=I^{\star_{J}}$ if and only if $I \subseteq \gamma^{-1} J$ for some $\gamma$ of valuation 0 ;

(d) if $I$ is nondivisorial, then $I$ is $\star_{\Delta}$-closed if and only if $I \subseteq \gamma^{-1} J$ for some $J \in \Delta$ and some $\gamma$ of valuation 0 .

Proof. (a) If $I$ is divisorial, then (since $I \neq R$ ) we must have $\left(R: M_{R}\right) \subseteq I$; in particular, $n-1 \in \mathbf{v}(I)$.

Suppose $n-1 \in \mathbf{v}(I)$; since $I$ contains every element of valuation at least $n$ (being $I T=I$ ), it contains also all elements of valuation $n-1$. Let $x$ be such that $\mathbf{v}(x)=n-1$ : then, $\mathbf{v}(x+r) \geq n-1$ for every $r \in V$, and thus $x+I \subseteq I$. Hence, $I$ is divisorial by [1, Proposition II.1.23].

(b) Let $L:=I \cup\{x \mid \mathbf{v}(x) \geq n-1\}$. If $n-1 \in \mathbf{v}(I)$, then $L=I$ and $I^{v}=L$ by the previous point. If $n-1 \notin \mathbf{v}(I)$, then (since $I$ contains any element of valuation at least $n), L$ is a fractional ideal of $R$ such that $\mathbf{v}(L)=\mathbf{v}(I) \cup\{n-1\}$; hence, it is divisorial and $\ell(L / I)=1$. It follows that $L=I^{v}$, as claimed. 
(c) Suppose $I \subseteq \gamma^{-1} J$, where $\mathbf{v}(\gamma)=0$. Since $J$ is not divisorial, $n-1 \notin \mathbf{v}(J)=$ $\mathbf{v}\left(\gamma^{-1} J\right)$; hence, using the previous point, $I=I^{v} \cap \gamma^{-1} J$ is closed by $\star_{J}$.

Conversely, suppose $I=I^{\star J}$. Since $I$ is nondivisorial, there must be $\gamma \in(I: J)$, $\gamma \neq 0$ such that $I \subseteq \gamma^{-1} J$ and $I^{v} \nsubseteq \gamma^{-1} J$. If $\mathbf{v}(\gamma)>0$, then $\gamma^{-1} J$ contains the elements of valuation $n-1$; it follows that $I^{v} \subseteq \gamma^{-1} J$ and thus that $I^{v} \subseteq I^{\star_{J}}$, against $I=I^{{ }_{J}}$. Hence, $\mathbf{v}(\gamma)=0$, as claimed.

(d) If $I \subseteq \gamma^{-1} J$ for some $J \in \Delta$ and some $\gamma$ such that $\mathbf{v}(\gamma)=0$, then $I^{\star \Delta} \subseteq$ $I^{\star_{J}}=I$, and thus $I$ is $\star_{\Delta}$-closed.

Conversely, suppose $I=I^{\star \Delta}$. For every $J \in \Delta$, the ideal $I^{\star_{J}}$ is contained in $I^{v}=I \cup\{x \mid \mathbf{v}(x) \geq n-1\}$; since $\ell\left(I^{v} / I\right)=1$, it follows that $I^{{ }_{J}}$ is either $I$ or $I^{v}$. Since $I=I^{\star \Delta}$, it must be $I^{\star J}=I$ for some $J$; by the previous point, $I \subseteq \gamma^{-1} J$ for some $\gamma$, as claimed.

An important consequence of the previous proposition is the following: suppose that $\Delta$ is a set of nondivisorial ideals in $\mathcal{F}_{0}(R)$ such that, when $I \neq J$ are in $\Delta$, then $I \nsubseteq \gamma^{-1} J$ for all $\gamma$ having valuation 0 . Then, for every subset $\Lambda \subseteq \Delta$, the set of ideals of $\Delta$ that are $\star_{\Lambda}$-closed is exactly $\Lambda$; in particular, each nonempty subset of $\Delta$ generates a different star operation.

We will use this observation to estimate the cardinality of $\operatorname{Star}(R)$ when the residue field is finite.

Proposition 3.3. Let $R$ be a Kunz domain such that $\mathbf{v}(R)=\langle n, n+1, \ldots, 2 n-$ $3,2 n-1\rangle$, and suppose that the residue field of $R$ has cardinality $q<\infty$. Then,

$$
|\operatorname{Star}(R)| \geq 2^{\frac{q^{n-2}-1}{q-1}} \geq 2^{q^{n-3}} .
$$

Proof. Let $L:=\{x \in V \mid \mathbf{v}(x) \geq n\}$; then, $A:=V / L$ is a $K$-algebra. Let $e_{1}$ be an element of valuation 1 , and let $e_{i}:=e_{1}^{i}$; then, $\left\{1=e_{0}, e_{1}, \ldots, e_{n-1}\right\}$ projects to a $K$-basis of $A$, which for simplicity we still denote by $\left\{e_{0}, \ldots, e_{n-1}\right\}$. The vector subspace spanned by $e_{0}$ is exactly the field $K$.

Since $V$ and $L$ are stable by multiplication by every element of valuation 0 , asking if $\gamma I \subseteq J$ for some $I, J \in \mathcal{F}_{0}(R)$ and some $\gamma$ is equivalent to asking if there is a $\bar{\gamma} \in A$ of "valuation" 0 such that $\bar{\gamma} \subseteq \bar{J}$, where $\bar{I}$ and $\bar{J}$ are the images of $I$ and $J$, respectively, in $A$. Hence, instead of working with ideals in $\mathcal{F}_{0}(R)$ we can work with vector subspaces of $A$ containing $e_{0}$.

Furthermore, if $V$ is a vector subspace of $A$ and $\gamma$ has valuation 0 , then $\gamma V$ has the same dimension of $V$; thus, if $V$ and $W$ have the same dimension, $\gamma V \subseteq W$ if and only if $\gamma V=W$. Let $\sim$ denote the equivalence relation such that $V \sim W$ if and only if $\gamma V=W$ for some $\gamma$ of valuation 0 . 
Let $X$ be the set of 2-dimensional subspaces of $A$ that contain $e_{0}$ but not $e_{n-1}$. Then, the preimage of every element of $X$ is an element of $\mathcal{F}_{0}(R)$ that does not contain any element of valuation $n-1$, and thus it is nondivisorial by Proposition $3.2(\mathrm{~b})$.

An element of $X$ is in the form $\left\langle e_{0}, \lambda_{1} e_{1}+\cdots+\lambda_{n-1} e_{n-1}\right\rangle$, where at least one among $\lambda_{1}, \ldots, \lambda_{n-2}$ is not 0 ; since $\left\langle e_{0}, f\right\rangle=\left\langle e_{0}, \lambda f\right\rangle$ for all $\lambda \in K, \lambda \neq 0$, there are exactly $\left(q^{n-1}-q\right) /(q-1)$ such subspaces.

Let $V \in X$, say $V=\left\langle e_{0}, f\right\rangle$, and consider the equivalence class $\Delta$ of $V$ with respect to $\sim$. Then, $W \in \Delta$ if and only if $\gamma W=V$ for some $\gamma$; since $1 \in W$, it follows that such a $\gamma$ must belong to $V$. Since $\gamma$ has valuation 0 , it must be in the form $\lambda_{0} e_{0}+\lambda_{1} f$ with $\lambda_{0} \neq 0$; furthermore, if $\gamma^{\prime}=\lambda \gamma$ then $\gamma^{-1} V=\gamma^{\prime-1} W$. Hence, the cardinality of $\Delta$ is at most $\frac{q^{2}-q}{q-1}=q$.

Therefore, $X$ contains elements belonging to at least

$$
\frac{1}{q} \frac{q^{n-1}-q}{q-1}=\frac{q^{n-2}-1}{q-1} \geq q^{n-3}
$$

equivalence classes; let $X^{\prime}$ be a set of representatives of such classes, and let $Y$ be the preimage of $X^{\prime}$ in the power set of $\mathcal{F}_{0}(R)$. Then, every subset of $Y$ generates a different star operation (with the empty set corresponding to the $v$-operation); it follows that

$$
|\operatorname{Star}(R)| \geq 2^{\frac{q^{n-2}-1}{q-1}} \geq 2^{q^{n-3}}
$$

as claimed.

For $n=4$, we can even calculate $|\operatorname{Star}(R)|$.

Proposition 3.4. Let $R$ be a Kunz domain such that $\mathbf{v}(R)=\langle 4,5,7\rangle$, and suppose that the residue field of $R$ has cardinality $q<\infty$. Then, $|\operatorname{Star}(R)|=2^{2 q}+3$.

Proof. Consider the same setup of the previous proof. We start by claiming that two vector subspaces $W_{1}, W_{2}$ of $A$ of dimension 3 that contain $e_{0}$ but not $e_{3}$ are equivalent under $\sim$.

Indeed, any such subspace must have a basis of the form $\left\{e_{0}, e_{1}+\theta_{1} e_{3}, e_{2}+\theta_{2} e_{3}\right\}$, and different pairs $\left(\theta_{1}, \theta_{2}\right)$ induce different subspaces; let $W\left(\theta_{1}, \theta_{2}\right):=\left\langle e_{0}, e_{1}+\right.$ $\left.\theta_{1} e_{3}, e_{2}+\theta_{2} e_{3}\right\rangle$. To show that two such subspaces are equivalent, we prove that they are all equivalent to $W(0,0)$. Let $\gamma:=e_{0}-\theta_{2} e_{1}-\theta_{1} e_{2}$ : we claim that $\gamma W\left(\theta_{1}, \theta_{2}\right)=W(0,0)$. Indeed, $\gamma e_{0}=\gamma \in W(0,0)$; on the other hand,

$$
\gamma\left(e_{1}+\theta_{1} e_{3}\right)=e_{1}+\theta_{1} e_{3}-\theta_{2} e_{2}-\theta_{1} e_{3}=e_{1}-\theta_{2} e_{2} \in W(0,0),
$$

and likewise

$$
\gamma\left(e_{2}+\theta_{2} e_{3}\right)=e_{2}+\theta_{2} e_{3}-\theta_{2} e_{3}=e_{2} \in W(0,0) .
$$


Hence, $W\left(\theta_{1}, \theta_{2}\right) \sim W(0,0)$.

Consider now the set $\Delta$ of nondivisorial ideals in $\mathcal{F}_{0}(R)$. By Lemma 2.5 and Proposition 3.2, $\Delta$ is equal to the union of the set $\mathcal{C}$ of the canonical ideals and the set $\mathcal{G}$ of the $I \in \mathcal{F}_{0}(R)$ such that $I T=I$. By Lemma 3.1 and Proposition 3.2, the elements of the latter correspond to the subspaces of $V / T$ containing $e_{0}$ but not $e_{3}$ : hence, we can write $\mathcal{G}=\mathcal{G}_{1} \cup \mathcal{G}_{2} \cup \mathcal{G}_{3}$, where $\mathcal{G}_{i}$ contains the ideals of $\mathcal{G}$ corresponding to subspaces of dimension $i$.

Given $\star \in \operatorname{Star}(R)$, let $\Delta(\star):=\left\{I \in \Delta \mid I=I^{\star}\right\}$. We claim that $\Delta(\star)$ is one of the following:

- $\Delta$

- $\Delta \backslash \mathcal{C}$

- $\Lambda \cup\{T\}$ for some $\Lambda \subseteq \mathcal{G}_{2}$;

- the empty set.

By Proposition 2.4, if $T \neq T^{\star}$ (i.e., if $T \notin \Delta(\star)$ ) then $\star=v$, and $\Delta(\star)=\emptyset$.

If $\Delta(\star)$ contains a canonical ideal then $\star$ is the identity, and thus $\Delta(\star)=\Delta$.

If $I$ is $\star$-closed for some $I \in \mathcal{G}_{3}$, but no canonical ideal is $\star$-closed, then every element of $\mathcal{G}_{3}$ must be closed, since any other $I^{\prime} \in \mathcal{G}_{3}$ is in the form $\gamma I$ for some $\gamma$ of valuation 0 (by the first part of the proof); furthermore, every element of $\mathcal{G}_{2}$ is the intersection of the elements of $\mathcal{G}_{3}$ containing it, and thus it is $\star$-closed. It follows that $\Delta(\star)=\Delta \backslash \mathcal{C}$; in particular, there is only one such star operation.

Let $\star$ be any star operation different from the three above. Then, $\Delta(\star)$ must contain $T$ and cannot contain any canonical ideal nor any element of $\mathcal{G}_{3}$. Hence, $\Delta(\star)$ must be equal to $\Lambda \cup\{T\}$ for some $\Lambda \subseteq \mathcal{G}_{2}$. Moreover, $\Lambda \cup\{T\}$ is equal to $\Delta(\star)$ for some $\star$ if and only if $\Lambda$ is the (possibly empty) union of equivalence classes under $\sim$. It follows that $|\operatorname{Star}(R)|=2^{x}+3$, where $x$ is the number of such equivalence classes.

By the proof of Proposition 3.3, the image of an element of $\mathcal{G}_{2}$ is in the form $\left\langle e_{0}, f\right\rangle$, where $f=\lambda_{1} e_{1}+\lambda_{2} e_{2}+\lambda_{3} e_{3}$ with at least one between $\lambda_{1}$ and $\lambda_{2}$ nonzero. Let $V\left(\lambda_{1}, \lambda_{2}, \lambda_{3}\right)$ denote the subspace $\left\langle e_{0}, f\right\rangle$; clearly, $V\left(\lambda_{1}, \lambda_{2}, \lambda_{3}\right)=$ $V\left(c \lambda_{1}, c \lambda_{2}, c \lambda_{3}\right)$ for every $c \in K \backslash\{0\}$. The subspaces equivalent to $V$ must have the form $\left(e_{0}+\theta f\right)^{-1} V$ for some $\theta \in K$, and, by using the basis $\left\{e_{0}, e_{0}+\theta f\right\}$ of $V$, we see that $\left(e_{0}+\theta f\right)^{-1} V\left(\lambda_{1}, \lambda_{2}, \lambda_{3}\right)=\left\langle e_{0},\left(e_{0}+\theta f\right)^{-1}\right\rangle$. If $\theta=0$, then $e_{0}+\theta f=e_{0}$, and thus $\left(e_{0}+\theta f\right)^{-1} V\left(\lambda_{1}, \lambda_{2}, \lambda_{3}\right)=V\left(\lambda_{1}, \lambda_{2}, \lambda_{3}\right)$; suppose, from now on, that $\theta \neq 0$. 
To calculate $\left(e_{0}+\theta f\right)^{-1}=e_{0}+\alpha_{1} e_{1}+\alpha_{2} e_{2}+\alpha_{3} e_{3}$, we can simply expand the product $\left(e_{0}+\theta f\right)\left(e_{0}+\alpha_{1} e_{1}+\alpha_{2} e_{2}+\alpha_{3} e_{3}\right)$, using $e_{i}=0$ for $i>3$; we obtain

$$
\left\{\begin{array}{l}
\alpha_{1}=-\theta \lambda_{1} \\
\alpha_{2}=-\theta\left(\lambda_{1} \alpha_{1}+\lambda_{2}\right) \\
\alpha_{3}=-\theta\left(\lambda_{1} \alpha_{2}+\lambda_{2} \alpha_{1}+\lambda_{3}\right) .
\end{array}\right.
$$

Since $\theta \neq 0$, the set $\left\{e_{0},\left(e_{0}+\theta f\right)^{-1}-e_{0}\right\}$ is a basis of $\left(e_{0}+\theta f\right)^{-1} V\left(\lambda_{1}, \lambda_{2}, \lambda_{3}\right)$; hence, $\left(e_{0}+\theta f\right)^{-1} V\left(\lambda_{1}, \lambda_{2}, \lambda_{3}\right)=V\left(\alpha_{1}, \alpha_{2}, \alpha_{3}\right)$. We distinguish two cases.

If $\lambda_{1}=0$, then $\lambda_{2} \neq 0$, and so we can suppose $\lambda_{2}=1$. Then, we have

$$
\left\{\begin{array}{l}
\alpha_{1}=0 \\
\alpha_{2}=-\theta \\
\alpha_{3}=-\theta \lambda_{3}
\end{array}\right.
$$

and so $\left(e_{0}+\theta f\right)^{-1} V\left(0,1, \lambda_{3}\right)=V\left(0,-\theta,-\theta \lambda_{3}\right)=V\left(0,1, \lambda_{3}\right)$ since $\theta \neq 0$. It follows that the only subspace equivalent to $V\left(0,1, \lambda_{3}\right)$ is $V\left(0,1, \lambda_{3}\right)$ itself; since we have $q$ choices for $\lambda_{3}$, this case gives $q$ different equivalence classes.

If $\lambda_{1} \neq 0$, we can suppose $\lambda_{1}=1$. Then, we get

$$
\left\{\begin{array}{l}
\alpha_{1}=-\theta \\
\alpha_{2}=-\theta\left(\alpha_{1}+\lambda_{2}\right)=-\theta\left(-\theta+\lambda_{2}\right) \\
\alpha_{3}=-\theta\left(-\theta\left(-\theta+\lambda_{2}\right)-\theta \lambda_{2}+\lambda_{3}\right) .
\end{array}\right.
$$

Since $\theta \neq 0$, we can divide by $-\theta$, obtaining

$$
\left(e_{0}+\theta f\right)^{-1} V\left(1, \lambda_{2}, \lambda_{3}\right)=V\left(1,-\theta+\lambda_{2}, \theta^{2}-2 \theta \lambda_{2}+\lambda_{3}\right) .
$$

Since $-\theta+\lambda_{2} \neq-\theta^{\prime}+\lambda_{2}$ if $\theta \neq \theta^{\prime}$, we have $\left(e_{0}+\theta f\right)^{-1} V\left(1, \lambda_{2}, \lambda_{3}\right) \neq\left(e_{0}+\right.$ $\left.\theta^{\prime} f\right)^{-1} V\left(1, \lambda_{2}, \lambda_{3}\right)$ for all $\theta \neq \theta^{\prime}$; thus, every equivalence class is composed by $q$ subspaces. Since there are $q^{2}$ such subspaces, we get $q$ additional equivalence classes.

Therefore, $\mathcal{G}_{2}$ is partitioned into $2 q$ equivalence classes, and so $|\operatorname{Star}(R)|=2^{2 q}+3$, as claimed.

Remark 3.5. (1) The estimate obtained in Proposition 3.3 grows very quickly; for example, if $q$ is fixed, it follows that the double logarithm of $|\operatorname{Star}(R)|$ grows (at least) linearly in $n=\ell(V / R)+1$. This should be compared with [8, Theorem $3.21]$, where the authors analyzed a case where the growth of $|\operatorname{Star}(R)|$ was linear in $\ell(\bar{R} / R)$ (where $\bar{R}$ is the integral closure of $R$, which in this case is nonlocal). 
(2) Let $V=K[[X]]$ be the ring of power series and consider the case $n=4$. Then, $T=K+X^{4} K[[X]]$, and using Theorem 2.9 and Proposition 3.3, we have the lower bound $|\operatorname{Star}(T)| \geq 2^{2 q}+q+2$. This estimate is not very far from the precise counting $|\operatorname{Star}(T)|=2^{2 q+1}+2^{q+1}+2$ obtained in [15, Corollary 4.1.2].

\section{References}

[1] V. Barucci, D. E. Dobbs and M. Fontana, Maximality properties in numerical semigroups and applications to one-dimensional analytically irreducible local domains, Mem. Amer. Math. Soc., 125(598) (1997), x+78 pp.

[2] H. Bass, On the ubiquity of Gorenstein rings, Math. Z., 82 (1963), 8-28.

[3] E. G. Houston, A. Mimouni and M. H. Park, Integral domains which admit at most two star operations, Comm. Algebra, 39(5) (2011), 1907-1921.

[4] E. G. Houston, A. Mimouni and M. H. Park, Noetherian domains which admit only finitely many star operations, J. Algebra, 366 (2012), 78-93.

[5] E. G. Houston, A. Mimouni and M. H. Park, Integrally closed domains with only finitely many star operations, Comm. Algebra, 42(12) (2014), 5264-5286.

[6] E. Houston, A. Mimouni and M. H. Park, Star operations on overrings of Noetherian domains, J. Pure Appl. Algebra, 220(2) (2016), 810-821.

[7] E. Houston, A. Mimouni and M. H. Park, Star operations on overrings of Prüfer domains, Comm. Algebra, 45(8) (2017), 3297-3309.

[8] E. G. Houston and M. H. Park, A characterization of local Noetherian domains which admit only finitely many star operations: The infinite residue field case, J. Algebra, 407 (2014), 105-134.

[9] J. Jäger, Längenberechnung und kanonische Ideale in eindimensionalen Ringen, Arch. Math. (Basel), 29(5) (1977), 504-512.

[10] E. Kunz, The value-semigroup of a one-dimensional Gorenstein ring, Proc. Amer. Math. Soc., 25 (1970), 748-751.

[11] E. Kunz, Beispiel: Die kanonische Idealklasse eines eindimensionalen CohenMacaulay-Rings, 103, Lecture Notes in Math., Vol. 238, Springer, Berlin, (1971), 17-24.

[12] T. Matsuoka, On the degree of singularity of one-dimensional analytically irreducible Noetherian local rings, J. Math. Kyoto Univ., 11 (1971), 485-494.

[13] J. C. Rosales and P. A. García-Sánchez, Numerical Semigroups, Developments in Mathematics, 20, Springer, New York, 2009.

[14] D. Spirito, Star operations on numerical semigroups, Comm. Algebra, 43(7) (2015), 2943-2963. 
[15] B. White, Star Operations and Numerical Semigroup Rings, Ph.D. thesis, The University of New Mexico, 2014.

\section{Dario Spirito}

Dipartimento di Matematica e Fisica

Università degli Studi "Roma Tre"

Roma, Italy

email: spirito@mat.uniroma3.it 